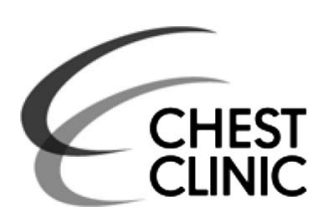
Medicine, University Hospital 12 de Octubre, Madrid, Spain 2Department of Cardiology, University Hospital 12 de Octubre, Madrid, Spain ${ }^{3}$ Department of Respiratory and Critical Care Medicine, OttoWagner-Spital, Vienna, Austria ${ }^{4}$ Department of Thoracic Surgery, University Hospital 12 de Octubre, Madrid, Spain

\section{Correspondence to}

Antolina Gómez López, Department of Respiratory Medicine, University Hospital 12 de Octubre, Madrid, Spain; antolinagl@hotmail.com

Received 19 October 2016 Revised 22 November 2016 Accepted 30 November 2016 Published Online First 2 January 2017

\section{CrossMark}

To cite: Gómez López A García Luján R, De Pablo Gafas A, et al. Thorax 2017;72:668-670.

\title{
First use of Amplatzer device for bronchopleural fistula after lung transplantation
}

\author{
Antolina Gómez López, ${ }^{1}$ Ricardo García Luján, ${ }^{1}$ Alicia De Pablo Gafas, ${ }^{1}$ \\ Felipe Hernández Hernández, ${ }^{2}$ Victoria Villena Garrido, ${ }^{1}$ Arschang Valipour, ${ }^{3}$ \\ Jose Carlos Meneses Pardo, ${ }^{4}$ Eduardo De Miguel Poch ${ }^{1}$
}

\section{INTRODUCTION}

Lung transplant recipients have a higher morbidity and mortality when compared with other solid organ transplants.

Particularly vascular complications can compromise the function of the transplanted lung and increase mortality. While vascular anastomotic stenosis is less common than central airways complications, it is associated with a high mortality rate if left untreated.

Here we describe a non-surgical treatment for a bronchopleural fistula due to a vascular complication after lobectomy.

\section{CASE REPORT}

A 49-year-old woman with emphysema secondary to $\alpha-1$ antitrypsin deficiency was admitted for evaluation of lung transplantation, due to progressive worsening of disease despite optimal medical management, including monthly intravenous prolastin therapy, maximum bronchodilator therapy and oxygen.

Based on the hospital protocol and consistent with recommended guidelines double lung transplantation was performed in June 2010. Surgery was complicated by an intraoperative injury of the left pulmonary artery, which required a cardiopulmonary bypass for 4 hours. During the following 48 hours the patient required vasoactive drugs (norepinephrine $0.2 \mu \mathrm{g} / \mathrm{kg} / \mathrm{min}$ and dobutamine $2 \mu \mathrm{g} /$ $\mathrm{kg} / \mathrm{min}$ ) and mechanical ventilation for 7 days (tracheostomy was performed on the 3rd day). Twenty-four hours after lung transplantation a chest radiograph showed a right basal infiltrate. On computed axial tomography an oedema and congestion in the right lower lobe was diagnosed. A subsequent transoesophageal echocardiogram demonstrated absence of flow in the right inferior pulmonary vein without evidence of a thrombotic occlusion. The decision of immediate right lower lobectomy (RLL) was made, followed by an uneventful immediate postoperative course.

A week after lobectomy (9th day after transplantation) a bronchopleural fistula was diagnosed clinically and confirmed endoscopically.

Bronchoscopy revealed a $9 \mathrm{~mm}$ dehiscence in the stump of the right lower lobe (figure 1), the transplantation sutures were intact.

Due to the complicated disease course and subsequent high perioperative risk in case of repeat thoracotomy, there was a need for an alternative, less-invasive technique to treat the bronchopleural fistula.

Repeated attempts to close the fistula by various endoscopic techniques including cyanoacrylate instillation (figure 2) and application of fibrin glue were not successful in closing the fistula.

During a multidisciplinary meeting the closure of the fistula with an Amplatzer device was suggested. The patient was informed about the novelty of the case, benefits and risks, and signed an informed consent.

Placement of the Amplatzer septal occluder device (ASOD) was performed under conscious sedation (fentanyl and midazolam) using videobronchoscopic guidance through the tracheostomy. The appropriate size of the ASOD was measured using a sizing catheter under fluoroscopy. The bronchial fistula was $9.3 \mathrm{~mm}$ in greatest diameter thus requiring an ASOD number 10 (10 $\mathrm{mm}$ waist diameter, $18 \mathrm{~mm}$ length, distal disc $22 \mathrm{~mm}$, proximal disc $18 \mathrm{~mm}$ diameter) (figure 3 ). The procedure lasted overall $45 \mathrm{~min}$, total fluoroscopy time was $5.4 \mathrm{~min}$. Postinterventional recovery was uneventful, the patient was discharged 18 days after the procedure (28 days after lung transplant) but the pleural cavity was colonised and infected by sensitive Pseudomonas aeruginosa and the chest tube was kept in place for 21 months until the pleural cavity was without signs of infection. The immunosuppressive medication regimen which the patient received after transplant was tacrolimus $0.1-0.3 \mathrm{mg} / \mathrm{kg} / \mathrm{day}$ (with target plasma levels of $10-15 \mathrm{ng} / \mathrm{mL}$ ), azathioprine $2 \mathrm{mg} / \mathrm{kg} /$ day: $100 \mathrm{mg} /$ 12 hours and prednisone $7.5 \mathrm{mg} / 12$ hours.

After ASOD implantation the patient underwent close clinical and endoscopic follow-up for 1 year and then at increasing time intervals, a follow-up CT (figure 4) at 72 months showed a residual pleural cavity on the right side without clinical signs of complications.

The last follow-up was performed 6 years after the intervention with no evidence of intercurrent infections or relevant local side effects.

Currently, she is 55 years old, with stable lung function $\left(\mathrm{FEV}_{1} 1700 \mathrm{~mL}(100 \%)\right)$, without oxygen treatment and with a high quality of life and no evidence of organ rejection.

\section{DISCUSSION}

With continuing improvements in postoperative medical care the 1-year lung transplantation survival rate may be as high as $81 \%$ today. 


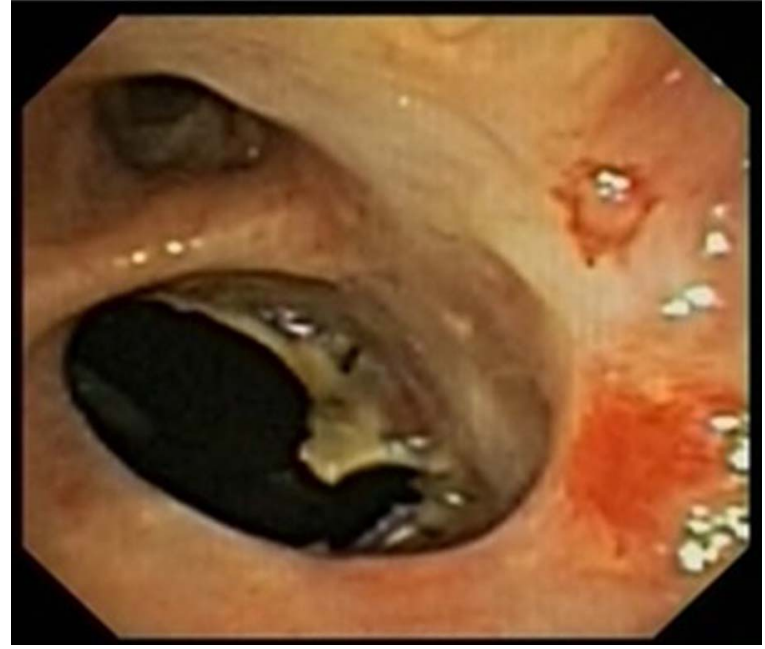

Figure 1 Bronchopleural fistula post lower right lobectomy (with metallic clips). Upper orifice; middle lobe.

Vascular complications are relatively uncommon after lung transplantation, with incidence rates reported as low as $1.75 \%$ for vascular anastomoses problems and approximately $1 \%$ for pulmonary venous obstruction stenosis. ${ }^{1}$ Donor-recipient size mismatch, surgical technique, and twisting, stricture and/or thrombosis of the pulmonary vessels have been cited as potential causes for the above with female patients and those with fibrotic lung disease being more susceptible to these complications.

A pulmonary venous obstruction should be suspected in patients with pulmonary oedema, unexplained hypoxaemia and frothy blood-stained secretion from the endotracheal tube. The differential diagnosis includes reperfusion oedema, myocardial dysfunction and primary graft dysfunction. Transoesophageal echocardiography with colour-flow Doppler imaging is diagnostic because it is considered an accurate guide to the morphological and functional state of the anastomoses and is extremely sensitive in detecting anatomical stenosis of the pulmonary veins. $^{2}$

Mild obstructions to flow may be treated without intervention but complete obstruction of a main pulmonary vein limits the therapeutic options to lobectomy or retransplantation. ${ }^{2}$

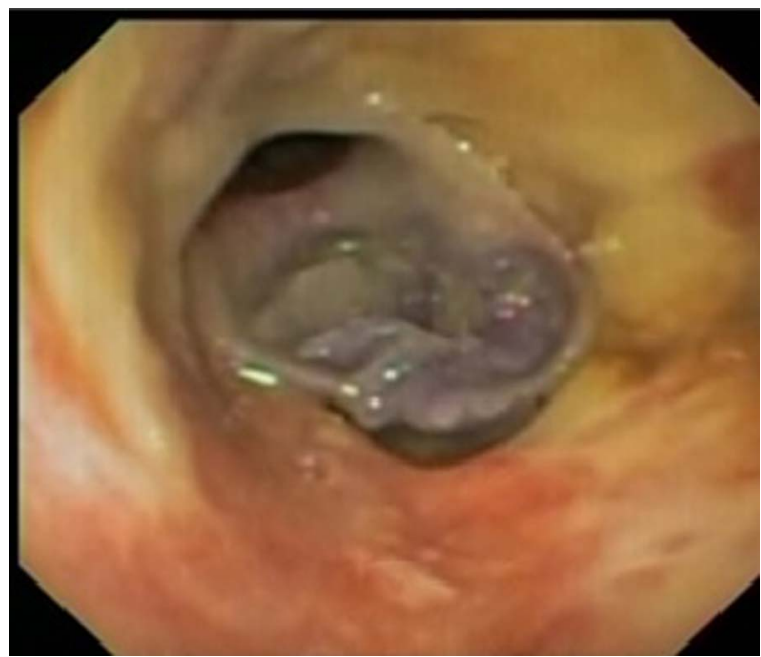

Figure 2 Fistula with cyanoacrylate.

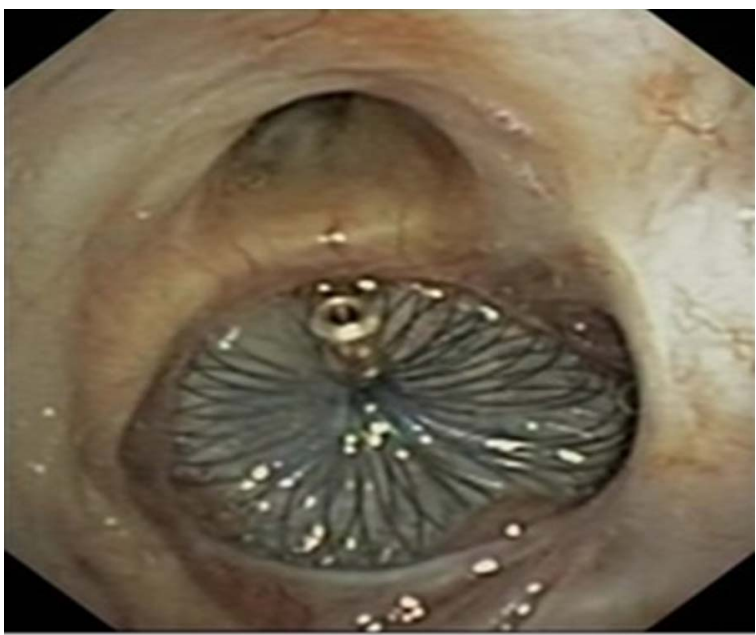

Figure 3 Fistula with Amplatzer septal occluder device.

Bronchopleural fistula is associated with high rates of morbidity and mortality. ${ }^{3}$

While surgical procedures are considered the standard care to treat bronchopleural fistula (BPF), an increasing number of patients are high-risk surgical candidates with underlying respiratory comorbidities or are in poor general condition. Thus, there is a need for minimally invasive treatment options. In this context, various endoscopic techniques, such as glues, coils and sealants, have previously been reported to treat patients with $\mathrm{BPF}$, with variable success rates reported in the literature. ${ }^{4}$ More recently, endoscopic unidirectional valve placement has been used in the treatment of prolonged BPF with a more consistent success rate. ${ }^{5}{ }^{6}$ While valve placement appears to be a safe and an effective potential alternative to surgical treatment, the majority of cases studied involved bronchoalveolar air leaks, rather than classic bronchopleural fistula.

Although the use of ASOD is best known for closing atrial septal defect in heart intervention it has been used for other pathologies such as tracheo-oesophageal fistulas and vascular fistulas in liver transplantation. Previous reports also suggested successful use of the ASOD for BPF after lobectomy and pneumonectomy.

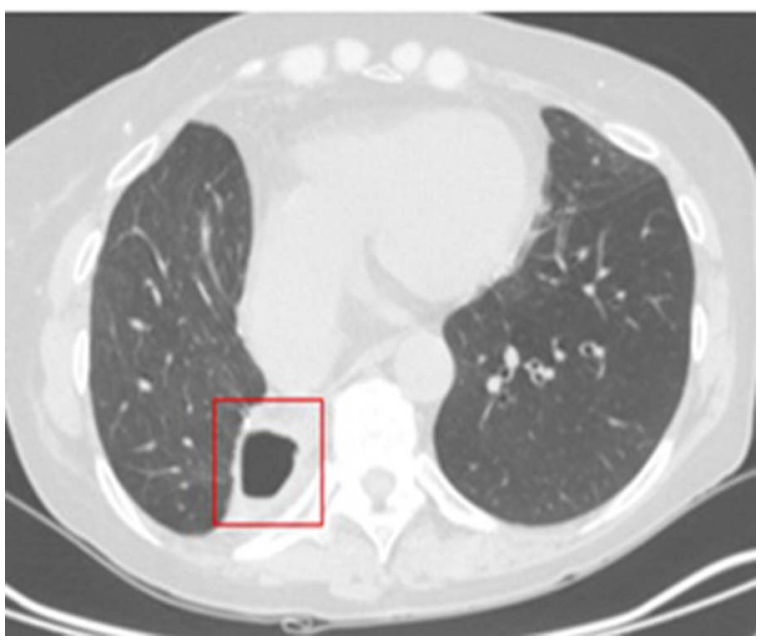

Figure 4 Residual pleural cavity after 6 years right lower lobectomy (red square). 
Fruchter et $a l^{7}$ reported long-term follow-up results up to 68 months in 31 surgical patients treated for BPF using this method. No mortality was observed during the immediate postprocedural period, however, short-term mortality ( $<30$ days) was $13 \%$ due to multiorgan failure and sepsis.

To the best of our knowledge the present case is the first report of ASOD in patients who developed a BPF after lung transplantation.

Bronchopleural fistula after lung transplantation in a postlobectomy lung graft is rare. The key points of BPF management consist of empyema drainage, infection control, fistula closure and pleural space obliteration. The choice of management depends on timing, size of the fistula, institutional expertise and the patient's general condition.

Chang et $a l^{8}$ previously reported on a patient who developed a BPF after bilateral lung transplantation for lymphangioleiomyomatosis. A stump dehiscence of $8 \times 4 \mathrm{~mm}$ was detected on bronchoscopy and successfully treated with endoscopic injection of tissue glue. In the present report, injection of fibrin glue did not result in BPF closure.

Brioude et $a l^{9}$ reported on a patient who underwent bilateral lung transplantation for Kartagener's syndrome, with a subsequent expansion deficit of the right lower lobe requiring a RLL. After 44 days the patient presented with acute respiratory distress syndrome due to a complete dehiscence of the lower bronchial suture with a multiloculated empyema at 30 days after lung transplantation. A right thoracotomy was performed for decortication and the visible BPF at the site of the bronchial suture was closed surgically. Due to the infection of the pleural cavity a thoracic window was performed, which was finally closed after 1 year without evidence of a BPF.

In the present report, surgery for the vascular complication was not performed as the patient was considered to be at high risk of subsequent postoperative complications. Thus, treatment with the ASOD was attempted as a minimal invasive alternative, and resulted in a good outcome.

In conclusion, bronchoscopy treatment of BPF with ASOD can be performed successfully in lung transplant patients, therefore offering an interesting treatment alternative in selected patients at high risk for thoracic surgery.

Competing interests None declared.

Patient consent Obtained.

Provenance and peer review Not commissioned; externally peer reviewed.

\section{REFERENCES}

1 Griffith BP, Magee MJ, Gonzalez IF. Anastomotic pitfalls in lung transplantation. J Thorac Cardiovasc Surg 1994;107:743-53; discussion 753-4.

2 Gonzalez-Fernández C, González-Castro A, Rodríguez-Borregán JC, et al. Pulmonary venous obstruction after lung transplantation. Diagnostic advantages of transesophageal echocardiography. Clin Transplant 2009;23:975-80.

3 Sirbu $\mathrm{H}$, Busch T, Aleksic I, et al. Bronchopleural fistula in the surgery of non-small cell lung cancer: incidence, risk factors, and management. Ann Thorac Cardiovasc Surg 2001;7:330-6.

4 Lois M, Noppen M. Bronchopleural fistulas: an overview of the problem with special focus on endoscopic management. Chest 2005;128:3955-65.

5 Firlinger I, Stubenberger E, Müller MR, et al. Endoscopic one-way valve implantation in patients with prolonged air leak and the use of digital air leak monitoring. Ann Thorac Surg 2013;95:1243-9.

6 Reed MF, Gilbert CR, Taylor MD, et al. Endobronchial valves for challenging air leaks Ann Thorac Surg 2015;100:1181-6.

7 Fruchter O, El Raouf BA, Abdel-Rahman N, et al. Efficacy of bronchoscopic closure of a bronchopleural fistula with Amplatzer devices: long-term follow-up. Respiration 2014;87:227-33.

8 Chang CC, Hsu HH, Kuo SW, et al. Bronchoscopic gluing for post-lung-transplant bronchopleural fistula. Eur J Cardiothorac Surg 2007;31:328-30.

9 Brioude G, Benoit D'journo X, Reynaud-Gaubert M, et al. Bronchial fistula after lobar size reduction for bilateral lung transplantation in Kartagener's syndrome: a surgical challenge. ICVTS 2013;17:184-6. 